\title{
Study and Analysis of Limit Cycle Bifurcation and Stability of Two Kinds of Dynamical Systems
}

\author{
Yao Haiyan \\ Liaocheng University Dongchang College, Liaocheng, Shandong, 252000, China \\ email: yaohaiyane@lcudcc.edu.cn
}

Keywords: Cubic Disturbance, Near Hamiltonian System, Homoclinic Ring

\begin{abstract}
Using the combination of qualitative analysis and numerical calculation, the limit cycle number and stability of the two mechanical systems are discussed. First of all, the limit cycles of the class of 2-order Hamiltonian functions are obtained by Melnikov bifurcation method. The system has four limit periods under three perturbations, three of which are generated near the homoclinic orbit. Then, the periodic vibration of the neural network model with non autonomous pulse delay is discussed. This pulse is differentiated by the principle of solution equivalence, and its equation is transformed into a continuous system. The existence and uniqueness of periodic solutions and exponential stability are obtained by using matrix spectrum theory and analytic inequality.
\end{abstract}

\section{Introduction}

The study of mechanics began at the end of the 19th century. Until 1960s, because of the development of differential geometry and differential topology, its theory had an important progress, and the value of the theory was controlled by the mathematics of mechanochemical ecology economics. By deepening people's understanding, his application prospects are very broad. It has become one of the powerful tools to study linear and nonlinear problems [1]. In other words, the dynamic system theory focuses on the global quality behavior of the system as it evolves over time. The research content mainly includes qualitative theory and stability theory. Plane qualitative theory mainly studies the phase of singularity and limit period. The general structure and properties of orbits in topological space.

\section{The Number of Limit Cycles for a Class of Quadratic Hamiltonian System with Cubic Perturbations}

One of the main problems in the qualitative theory of real machine plane system is the study of limit period, which includes the number position relationship between limit period and quantity stability. Generally speaking, it is difficult to find the limit cycle number for a given plane polynomial differential system. On the other hand, in the disturbance system near the center of the amorphous wafer, the occurrence of the limit period has many results [2]. The confusion in gay clinics has many consequences. For example, the non universal quadratic Hamiltonian function has two limit periods near the homocluting ring under quadratic perturbation. In this chapter, we investigate the class of cubic near Hamilton functions of quadratic systems whose non-interference systems are symmetric homoclinic rings. This system has four submission cycles. The conditions of four cycles are given. For the melniv function method of plane rings, according to the new conditions given by the plane system under the action of "multi parameters", it is investigated that the number 2 of homoclinic rings under the action of "any two times" of non permanent quadratic Hamiltonian system is proved. However, the number of rings of homoclinic rings for any non universal quadratic Hamiltonian system with three perturbations. No matter how many limit cycles are, there are three perturbations in the unconventional quadratic Hamilton - SevenOn system. Little has been achieved on this point. In this chapter, the second-order Hamiltonian system with three perturbations is considered to have four limit periods. It's very difficult. We just used special 
circumstances to confirm the correctness of the results [3]. Therefore, it is also meaningful to get general results. In addition, the limit period number of non ordinary quadratic hamll seven on system under high disturbance is more than 4 times.

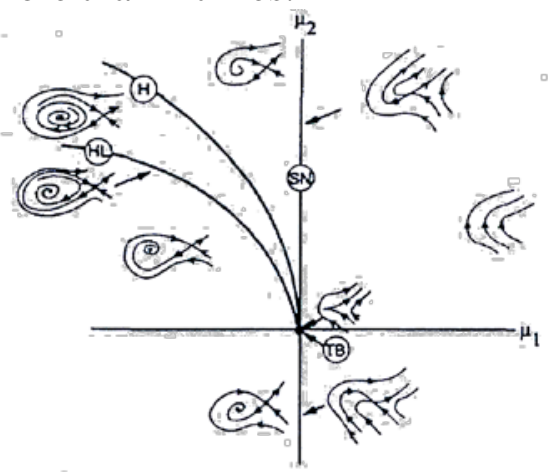

Figure 1 Bifurcation diagram and corresponding phase diagram of the system

\section{Periodic Oscillation of Neural Networks for BAM Model with Impulsive Delay}

Facts tell us that things depend not only on the present state, but also on the past state. In this case, it is reasonable to consider the delay differential equation. In order to study the exponential stability of the unilateral bilateral neural network model with time delay, the real behavior using Mmatrix and constant change formula is complex, and will change rapidly at a certain stage of development. This phenomenon is usually called pulse phenomenon [4]. The reason is that the description of the changing law of things has been successfully applied in many fields. In addition, chaos control and neural network for pattern recognition of parallel communication are also studied successfully. Therefore, it is of great significance to study the methods of dealing with the stability of the neural network model under the condition of pulse delay, including the richness of the results. In recent years, impulsive differential equations have been widely studied. Recently, the delay of signal transmission between axons (earthquakes) does not generate or generate two kinds of bidirectional conjectures. Their stability is widely studied in many fields, such as proposal, pattern recognition, automatic control and so on. Based on the analysis of unequal matrix theory and spectral theory, the existence, global charm and global exponential stability are obtained. In recent years, in order to oscillate the BAM neural network with certain coefficients or variable parameters periodically, some scholars have obtained many results, among which Lyapunov function is properly constructed, but it is not easy to find the appropriate Lyapunov function. The uniqueness of periodic solution, global charm and global exponential stability is obtained by using the analytic knowledge of inequality matrix theory and spectral theory. The activation function does not need to be differentiable or monotonic. We can consider more complex systems through related theorems and methods.

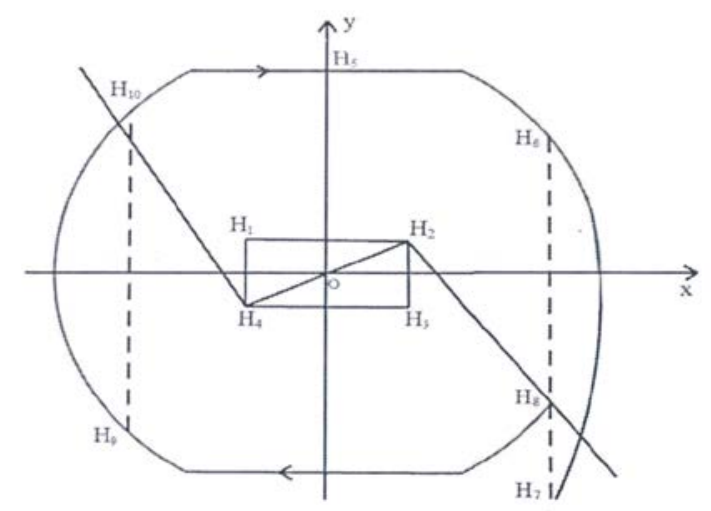

Figure 2 Structure of poincars Bendixson ring region 


\section{Dynamic Analysis of Memristor Model with Piecewise Function}

The main contents of this chapter are as follows: firstly, the memristor model with partial functions (3.1) is simply introduced, which simplifies the model as a system that simplifies the qualitative analysis and branch theory of dynamic system. Parameters are obtained in different ranges, and the number, types and stability of equilibrium points are studied in detail [5]. Three types of specific equilibrium points are obtained. Two Hopf bifurcation surfaces are obtained. These are the special properties of memristor with memory function. By using poincarg Bendixson ring domain theorem and constructing ring domain, it is proved that the system has inherent unstable periodic orbit. The simulation results show that when the system reaches a certain value, stable periodic orbit, charm invariant loop and alien diagnostic orbit are generated. All theoretical analyses are verified by numerical simulation. Phase diagrams of these parameters with different values

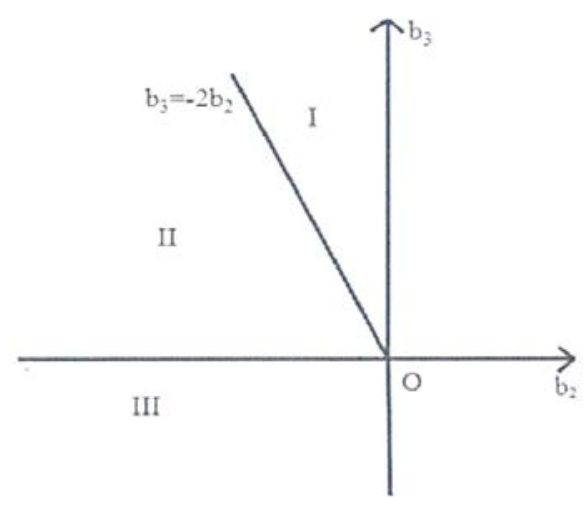

Figure 3 Parameter plan

\section{The Realization of Resistor Circuit Provides Some Theoretical Basis}

In this paper, the equilibrium problem of non hyperbolic system is studied. According to the central flow theorem, the purpose of maintaining the equilibrium of the system is restricted by the central flow form of the system. The stability of the point can be judged, but the central flow theorem cannot judge the type of equilibrium point [6]. By using the three theorems in the second chapter of Zhang et al. [2], the briot bouquet transformation and a series of topological transformations of the system are carried out to obtain a simplified system and judge the type and stability of the equilibrium point. Our results show that there are three types of singular point series in the resistance model with diffractive function, a unique unstable periodic orbit and a stable periodic orbit. These important dynamic phenomena are the typical characteristics of the difference between memristors and electronic devices without memory function. They are memristor circuits with segment function. It plays an important role in the actual electronic circuit. For this kind of two-dimensional resistance system, we only do some local research [7]. I hope you will continue this work in the future. For this system, we can study the boundary, global, periodic orbit branching and other issues, and more systematically study the theory of memory resistor system. Perfect.

\section{Dynamic Analysis of the Memristor Model of Diode}

The main contents of this chapter are as follows: firstly, the memristor mathematical model of nonlinear oscillator is obtained by replacing Cai - Shaotang diode, and the continuous twodimensional system is obtained by dimensionless processing. This paper mainly discusses the number, type and stability of system equilibrium points in different parameter regions, in which there are two branch behaviors of the system, i.e. branch and Hopf branch; these parameter regions have no periodic orbits. The qualitative analysis method and bifurcation theory of dynamic system 
are used to prove that the system has rich nonlinear dynamic behavior. When the system parameters take different values, the system will fork and branch [8]. The Soto mayor's theorem of the system whose branches are strictly proved is used. The system is transformed into a general Li 6nard system by using the concept of substitution. Hopf bifurcation is proved by using the conclusion and bifurcation theory of the General Li 6iard system.

\section{Conclusion}

With the rapid development of China's economy, high-speed rail has greatly shortened all travel time. High speed rail is more and more important to people's life. Bogey is one of the most important factors in the structure of railway vehicles [9]. The frame and some devices are similar in size to ordinary cars, and the EMU bracket can be rotated to a certain extent to this type of bogey. There are two bogeys under each bracket. Bogey is one of the main high-speed technologies of EMU, which plays the role of bearing, guiding, dumping, pulling bow, braking, etc. Whether the high-speed train can run or not is basically determined by bogey, which can also make the train stop at high speed. Bogey also has a basic brake. Therefore, the various parameters of the landing gear of the high-speed train directly determine the stability and comfort of the vehicle. In recent years, applications have used a variety of advanced technologies and installed new bogey to make it travel in a better environment. Now, a series of mathematical models are set up for bogey and body of high-speed train. When the parameters of these models change, the stability of the differential power system changes, which may lead to branching phenomenon [10]. These phenomena correspond to high-speed trains, which may cause severe shaking and derailment during train operation. Then, other phenomena, so the balance in Hopf bifurcation, and whether it can produce limit bicycle Hopf bifurcation, whether it can ignore these differential fields, different stability analysis, linear mechanics knowledge, hope to use. It is simple to provide theoretical support for bogey parameter value.

\section{References}

[1] Pegah Moghimi, Rasoul Asheghi, Rasool Kazemi. On the Number of Limit Cycles Bifurcated from a Near-Hamiltonian System with a Double Homoclinic Loop of Cuspidal Type Surrounded by a Heteroclinic Loop. International Journal of Bifurcation \& Chaos, vol. 28, no. 1, pp. 1850004, 2018.

[2] Pegah Moghimi, Rasoul Asheghi, Rasool Kazemi. On the Number of Limit Cycles Bifurcated from Some Hamiltonian Systems with a Double Homoclinic Loop and a Heteroclinic Loop. International Journal of Bifurcation \& Chaos, vol. 27, no. 4, 2017.

[3] Liangcheng Cai, Zhengyou He, Haitao Hu. A New Load Frequency Control Method of MultiArea Power System via the Viewpoints of Port-Hamiltonian System and Cascade System. IEEE Transactions on Power Systems, vol. 32, no. 3, pp. 1689-1700, 2017.

[4] Naiara V. de Paulo, Pedro A. S. Salomão. On the multiplicity of periodic orbits and homoclinics near critical energy levels of Hamiltonian systems in $\$ \backslash \backslash$ mathbb $\{\mathrm{R} \wedge 4$. Transactions of the American Mathematical Society, 2017.

[5] Feng Liang, Dechang Wang. Limit Cycle Bifurcations Near a Piecewise Smooth Generalized Homoclinic Loop with a Saddle-Fold Point. International Journal of Bifurcation \& Chaos, vol. 27, no. 5, pp. 1750071, 2017.

[6] Marzieh Mousavi, Hamid R. Z. Zangeneh. Limit Cycle Bifurcations by Perturbing a Hamiltonian System with a 3-Polycycle Having a Cusp of Order One or Two. International Journal of Bifurcation \& Chaos, vol. 28, no. 3, pp. 1850038, 2018.

[7] Xun Lei, Zenghui Gu, Jianbin Ma,. Investigation of the local dispersion change in anomalous dispersion microcavity and quantitative analysis of the phase-matching in Kerr comb generation. 
Applied Optics, vol. 56, no. 16, pp. , pp. 4828, 2017.

[8] Purnima Ghale, Harley T. Johnson. A sparse matrix-vector multiplication based algorithm for accurate density matrix computations on systems of millions of atoms. Computer Physics Communications, vol. 227, 2018.

[9] Gomez J A, Degroote M, Zhao J, et al. Spin polynomial similarity transformation for repulsive Hamiltonians: interpolating between coupled cluster and spin-projected unrestricted Hartree-Fock, vol. 19, 2017.

[10] Alessandro Fortunati, Stephen Wiggins. Normal forms à la Moser for aperiodically timedependent Hamiltonians in the vicinity of a hyperbolic equilibrium. Communications on Pure \& Applied Mathematics, vol. 9, no. 4, pp. 1109-1118, 2017. 